CARDIOVASCULAR MEDICINE

\title{
Long term follow up of radiofrequency catheter ablation of atrial flutter: clinical course and predictors of atrial fibrillation occurrence
}

\author{
E Bertaglia, F Zoppo, A Bonso, A Proclemer, R Verlato, L Corò, R Mantovan, D D’Este, F Zerbo, \\ P Pascotto, on behalf of the Northeastern Italian Study on Atrial Flutter Ablation Investigators
}

See end of article for authors' affiliations

Correspondence to:

Dr E Bertaglia, Via

Mariutto, 13, 30135-

Mirano (VE), Italy;

ulss 13 mirano.

elettrofisiologia@wind.

it.net

Accepted 19 June 2003
Objectives: To evaluate the time to onset and the predictors of atrial fibrillation (AF) during long term follow up of patients with typical atrial flutter (AFL) treated with transisthmic ablation.

Design: Prospective multicentre study.

Methods and results: 383 patients (75.4\% men, mean (SD) age 61.7 (11.1) years) who underwent transisthmic ablation for typical AFL were investigated. In 239 patients (62.4\%) AF was present before ablation. Ablation proved successful in 367 patients (95.8\%). During a mean (SD) follow up of 20.5 (12.4) months, $41.5 \%$ of patients reported AF. The cumulative probability of postablation AF increased continuously as time passed: it was $22 \%$ at six months, $36 \%$ at one year, $50 \%$ at two years, $58 \%$ at three years, and $63 \%$ at four years.

Conclusions: AF occurred in a large proportion of patients after transisthmic catheter ablation of typical AFL. The occurrence of AF was progressive during follow up. Preablation AF, age $<65$ years, and left atrial size $>50 \mathrm{~mm}$ are associated with postablation AF occurrence.
R adiofrequency catheter ablation targeting the isthmus between the tricuspid annulus and the inferior vena cava is an established treatment for typical atrial flutter (AFL). It is successful in more than $90 \%$ of patients. ${ }^{1-7}$

However, in the clinical setting, AFL and atrial fibrillation (AF) often coexist, and the follow up of patients successfully treated with transisthmic ablation is complicated by the occurrence of $\mathrm{AF}$ in $10-47 \%$ of patients. ${ }^{25-15}$ Indeed, although caused by different electrophysiological mechanisms, AFL and AF may share the same arrhythmogenic substrate. ${ }^{12} 1617$

Until the introduction of transisthmic catheter ablation, some questions were raised by cardiologists: is transisthmic ablation a definite cure for patients with AFL or would their follow up be characterised by the occurrence of other atrial arrhythmias? Should transisthmic ablation be suggested for patients with a history of both AFL and AF?

Many studies, some recent, have tried to answer these questions. ${ }^{2-15}$ 18-21 $^{1}$ However, most of these studies investigated few patients and the duration of the follow up was often short. Thus, there are no definitive answers.

The aim of this prospective multicentre study was to evaluate the time to onset and the predictors of postablation AF during long term follow up in a large cohort of patients with typical AFL treated with transisthmic radiofrequency catheter ablation.

\section{METHODS}

\section{Study population}

The Northeastern Italian study on atrial flutter ablation was a multicentre prospective study of typical AFL ablation conducted in six hospitals in the Northeast of Italy. The study group consisted of 383 consecutive patients who underwent radiofrequency catheter ablation for recurrent typical AFL from October 1996 to June 2001. Typical AFL was identified by negative flutter waves in the inferior leads and positive flutter waves in lead $\mathrm{Vl} .{ }^{20}$ Each patient still had AFL during pharmacological treatment with at least one antiarrhythmic drug (AAD). Previous episodes of AF were not considered to contraindicate ablation. Preablation AF was defined as the documentation during ECG or Holter ECG monitoring of at least one episode of AF lasting more than one minute.

Transthoracic two dimensional imaging and pulsed Doppler echocardiography were performed before ablation in all patients. Left ventricular ejection fraction was determined by calculating the end diastolic and end systolic volumes according to Folland and colleagues. ${ }^{22}$ Left atrial size was measured at end systole in the parasternal long axis view.

\section{Electrophysiological study and radiofrequency catheter ablation}

Written informed consent was obtained before transcatheter ablation from all patients. Ablation was performed in the fasting state after withdrawal of all $\mathrm{AAD}$ for at least five half lives (apart from amiodarone) in all patients without a history of AF and during treatment with class IC or III AADs in patients with previous documented episodes of AF. Anticoagulation was withdrawn two days before the procedure to obtain an international normalised ratio of $<2.0$ during the procedure. An initial bolus of heparin (5000 U) was administered after the insertion of catheters.

Simultaneous surface 12 lead ECGs and bipolar intracardiac ECGs were continuously acquired with a filter bandwidth of $30-500 \mathrm{~Hz}$, digitised, and displayed on a high resolution video monitor for inspection and subsequent review. Data were stored on optical disk for retrieval and offline analysis.

The procedures were performed in the following manner: four multipolar catheters were inserted from the right or left

Abbreviations: $A A D$, antiarrhythmic drug; $A F$, atrial fibrillation; $A F L$, atrial flutter 
femoral veins, the right internal jugular vein, the left subclavian vein, or the left brachial vein: one quadripolar catheter ( $5 \mathrm{~mm}$ interelectrode distance), positioned at the His bundle; one decapolar catheter $(2,5$, and $2 \mathrm{~mm}$ interelectrode distances), positioned in the coronary sinus, with the proximal electrode pair positioned at the ostium; a 20 electrode Halo catheter $(2,7$, and $2 \mathrm{~mm}$ intervals), positioned around the tricuspid valve to assess annular activation; and the ablation catheter.

Bidirectional isthmus conduction and collision of dual wave front were obtained. ${ }^{23}$

Ablation was performed during typical AFL (193 patients) or during continuous pacing from the low lateral right atrium or from the proximal coronary sinus (190 patients). Different ablation catheters were used: $4 \mathrm{~mm}$ distal tip (37 patients), $8 \mathrm{~mm}$ distal tip (317 patients), $10 \mathrm{~mm}$ distal tip (3 patients), and $4 \mathrm{~mm}$ distal irrigated tip (26 patients).

The ablation was anatomically guided. The end point was the achievement of a line of complete conduction block between the tricuspid valve and the inferior vena cava (posterior isthmus). If this attempt proved unsuccessful, another line of block was created between the septal portion of the tricuspid valve, the ostium of the coronary sinus, and the inferior vena cava (septal isthmus). ${ }^{23}$

Ablation was deemed successful if AFL terminated during radiofrequency delivery, AFL was no longer inducible, and bidirectional or unidirectional counterclockwise conduction block appeared..$^{6}$

If the first procedure was not successful, repeated catheter ablation was advised.

\section{Follow up}

After catheter ablation, all patients underwent continuous ECG monitoring for at least 24 hours before hospital discharge. Class I or III AADs were prescribed to all patients with previous concomitant AF. Aspirin 160 mg was administered for four weeks unless systemic anticoagulation with warfarin was indicated. In this case warfarin continued to be administered for at least four weeks.

Outpatient follow up, ECG, and Holter ambulatory ECG monitoring were performed after one month, three months, and at six month intervals thereafter. Patients were encouraged to contact one of the investigators at any time if they experienced recurrent palpitations.

\section{Statistical analysis}

Continuous variables are presented as mean (SD). Discrete variables are presented as percentages.

Variables were compared by Fisher's exact test for categorical variables and unpaired $t$ test for continuous variables. A probability value of $p<0.05$ was considered significant.

Among patients with successful ablation the actuarial probability of freedom from AF after ablation was calculated with the method of Kaplan and Meier. ${ }^{24}$ Differences between the curves were tested for significance by the log rank statistic. ${ }^{25}$ Twelve clinical and procedural variables were subjected to multivariate analysis with a Cox proportional hazards model and stepwise backward selection to identify significant and independent predictors of postablation AF. ${ }^{26}$ Variables in the initial model were: $(1)$ sex; $(2)$ age $(\leqslant 65$ years or $>65$ years); ( 3 ) duration of arrhythmic history ( $\leqslant 1$ year or $>1$ year); (4) duration of AFL episodes $(\leqslant 48$ hours or $>48$ hours); (5) preablation AF; (6) prevalence of AF in the arrhythmic history; (7) AF converted into AFL by oral class IC drugs (propafenone and flecainide) or amiodarone; (8) number of AAD previously tried $(\leqslant 2$ drugs or $>2$ drugs); (9) presence of structural heart disease; (10) left atrial size $(\leqslant 50 \mathrm{~mm}$ or $>50 \mathrm{~mm})$; (11) left ventricular ejection fraction ( $\leqslant 40 \%$ or $>40 \%$ ); and (12) creation of bidirectional transisthmic block. A significant increase in risk was obtained if the $95 \%$ confidence interval exceeded 1 and the probability value of the Wald test was $\mathrm{p}<0.05$.

Analysis was performed with SPSS (version 11.0, SPSS Inc, Chicago, Illinois, USA).

\section{RESULTS}

\section{Study population}

Table 1 summarises baseline patient characteristics. Structural heart disease was present in 182 of 383 patients (47.6\%). Hypertensive cardiopathy in 93 (24.3\%) and ischaemic heart disease in $47(12.3 \%)$ were the most frequent diseases. Two hundred and thirty nine of the 383 patients $(62.4 \%)$ had a history of AFL and AF. AF was the predominant arrhythmia in 134 patients $(34.0 \%)$. In 101 patients $(26.3 \%)$ class IC drugs (propafenone and flecainide) or amiodarone converted AF into AFL.

\section{Radiofrequency catheter ablation}

Among the 193 patients in whom the ablation was performed during AFL, the mean AFL cycle length was 253.4 (36.3) ms and a counterclockwise typical AFL was documented in 147 $(76.0 \%)$. Transisthmic ablation proved successful in 367 of 383 patients $(95.8 \%)$. A bidirectional conduction block was obtained in 333 of 383 patients $(86.9 \%)$ and a unidirectional counterclockwise block in 34 (8.9\%). Forty patients $(10.4 \%)$ needed two or more procedures to be successfully ablated.

The mean fluoroscopy time was 34.1 (21.4) minutes (range 7-120 minutes). The mean duration of radiofrequency application was 15.3 (9.7) minutes (range 3-56 minutes).

Six of 383 patients ( $1.6 \%$ ) had complications. Two patients developed complete atrioventricular block during septal isthmus ablation and needed a pacemaker. In one patient, sustained ventricular tachycardia developed during radiofrequency application at the edge of the tricuspid annulus. The arrhythmia was interrupted by external shock. A groin haematoma was reported in two patients and a thrombosis of the right femoral vein was reported in one patient.

\section{Follow up}

The mean follow up duration was 20.5 (12.4) months (range 6-55 months). Three of the 383 patients $(0.8 \%)$ died: in two patients death was non-cardiovascular and one had a fatal cerebral haemorrhage.

Recurrence of typical AFL was documented in 41 of 367 patients $(11.2 \%)$. In 31 patients, one or two repeated sessions of radiofrequency ablation definitively eliminated the AFL.

At the end of follow up, 201 of 367 patients (54.8\%) were treated with AADs: three patients $(0.8 \%)$ with class $1 \mathrm{~A}, 84$ patients $(22.9 \%)$ with class $1 C$, and 114 patients $(31.0 \%)$ with class III drugs.

\section{Occurrence of AF after transisthmic ablation}

Occurrence of AF after transisthmic ablation was evaluated in 357 patients. Patients with unsuccessful radiofrequency ablation and patients in whom AFL recurred without being

Table 1 Patient characteristics $(n=383)$

\begin{tabular}{ll}
\hline Age (years) & $61.7(11.1)$ \\
Male sex & $289(75.4 \%)$ \\
Symptom duration (years)) & $4.1(4.0)$ \\
Number of antiarrhythmic drugs & $1.9(1.0)$ \\
Preablation atrial fibrillation & $239(62.4 \%)$ \\
Structural heart disease & $182(47.6 \%)$ \\
Left ventricular ejection fraction (\%) & $58.9(9.7)$ \\
Left atrial size (mm) & $43.3(5.6)$
\end{tabular}

Data are mean (SD) or number (\%). 
treated again were excluded from the analysis. Follow up duration was six months for 357 of 357 patients (100.0\%), 12 months for $277(77.6 \%), 24$ months for 161 (45.1\%), 36 months for 65 (18.2\%), and 48 months for $24(6.7 \%)$.

$\mathrm{AF}$, defined as the documentation during ECG or ECG Holter monitoring of at least one minute of AF, occurred in 148 of 357 patients (41.5\%). AF occurrence increased progressively as time passed. The cumulative probability of postablation AF was $22 \%$ at six months, $36 \%$ at one year, $50 \%$ at two years, $58 \%$ at three years, and $63 \%$ at four years (fig 1).

Among the 148 patients in whom postablation AF was documented, $50(33.8 \%)$ were completely asymptomatic.

\section{Variables correlated with AF occurrence}

According to the results of multivariate analysis, a history of preablation AF (hazard ratio (HR) 2.818, 95\% confidence interval (CI) 1.703 to 4.661; $p<0.0001$ ), age $<65$ years (HR 1.464, 95\% CI 1.003 to $2.137 ; \mathrm{p}<0.05$ ), and left atrial size $>50 \mathrm{~mm}$ (HR 1.782, 95\% CI 1.020 to 3.112; p < 0.05) were significantly correlated with occurrence of postablation AF.

Preablation AF was present in 120 of 148 patients (81.1\%) with postablation AF and in 102 of 209 patients (48.8\%) without postablation AF. At four years, the probability of postablation $\mathrm{AF}$ was $68 \%$ for patients with preablation $\mathrm{AF}$ and $52 \%$ for patients without preablation AF $(\mathrm{p}<0.0001$, fig 2). Time to first AF occurrence was significantly shorter in patients with preablation AF than in patients without preablation $\mathrm{AF}$ (9.7 (5.3) months $v$ 18.1 (12.2) months, respectively; $\mathrm{p}<0.000001)$. This led to a different progression of AF between patients with and patients without preablation AF (fig 2). During the first two years, the rate of AF occurrence was very high $(66 \%)$ in patients with preablation $\mathrm{AF}$ and quite low (12\%) in patients without preablation AF.

The mean age was 60.2 (10.7) years in patients with postablation AF and 62.8 (11.4) years in patients without postablation AF. Preablation AF was documented in 144 of 221 patients $(65 \%)<65$ years old and in 95 of 162 patients $(59 \%) \geqslant 65$ years $(p=0.25)$. Similarly, predominant preablation AF occurred in 80 of 221 patients $(36 \%)<65$ years old and in 54 of 162 patients $(33 \%) \geqslant 65$ years old $(p=0.64)$. The mean duration of follow up in the successfully ablated patients was 20.9 (12.6) months in the younger patients and 20.0 (12.2) months in the older group

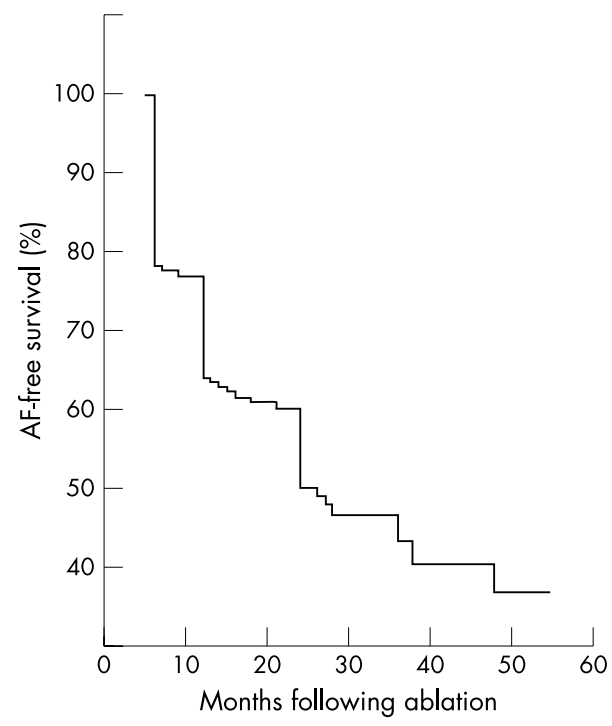

Figure 1 Kaplan-Meier estimate of the time to atrial fibrillation occurrence in the total population.

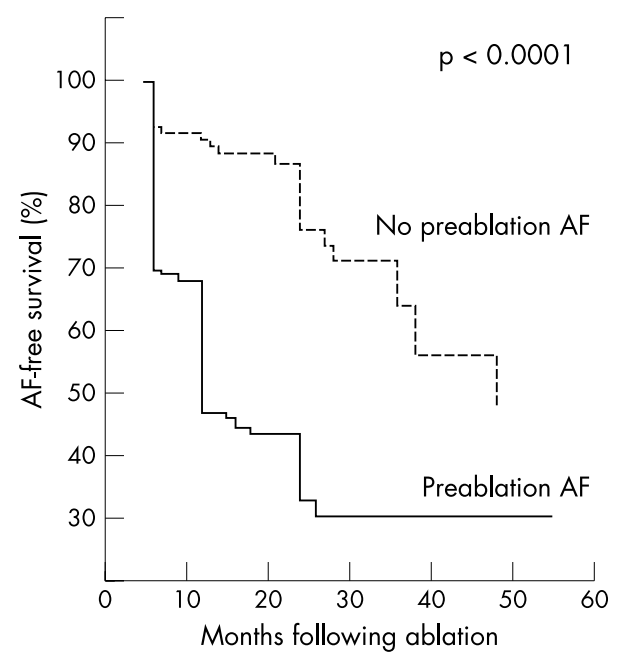

Figure 2 Kaplan-Meier estimates of the time to atrial fibrillation occurrence in the patients without preablation atrial fibrillation (dotted line) and in the patients with preablation atrial fibrillation (solid line).

$(p=0.17)$. At the end of the follow up, the rates of patients taking AAD were similar in the two groups (111 of 207 patients $(54 \%)<65$ years $v 79$ of 150 patients $(53 \%) \geqslant 65$ years; $\mathrm{p}=0.94)$.

The mean left atrial size was 44.1 (5.5) $\mathrm{mm}$ in patients with postablation $\mathrm{AF}$ and 43.0 (5.1) $\mathrm{mm}$ in patients without postablation AF.

\section{DISCUSSION}

\section{Main findings}

To the best of our knowledge this is the largest study to evaluate the long term results of transisthmic radiofrequency catheter ablation in patients with typical AFL with or without preablation AF. In this study AF occurred frequently despite the use of AAD; the occurrence of AF increased continuously during follow up; and a history of preablation $\mathrm{AF}$, age $<65$ years, and left atrial size $>50 \mathrm{~mm}$ were significantly correlated with occurrence of postablation AF.

\section{Occurrence of AF after transisthmic ablation}

As reported in previous papers, AF frequently occurs after transisthmic ablation of AFL, ranging from $12-54 \% .^{8-15} 2021$ Our data, obtained in a large cohort of patients, confirmed these results. Indeed, AF was observed in $41.5 \%$ of patients in our study a mean of 20.5 (12.4) months after ablation of AFL. Our population differed from those of previous studies in that it included more patients with preablation AF (62\%) and patients in whom AF was the predominant preablation arrhythmia.

The occurrence of AF increased progressively as time passed (fig l): at four years, the cumulative probability of AF occurrence had risen to $62 \%$. The progression of AF differed between patients with and patients without preablation $\mathrm{AF}$ (fig 2). While in the former group almost all of the recurrences of AF appeared during the first two years $(66 \%)$, in patients without preablation $\mathrm{AF}$ the rate of $\mathrm{AF}$ occurrence was quite low during the first two years (12\%) and increased significantly later $(52 \%$ at four years). In accordance with this observation, the time to first $\mathrm{AF}$ occurrence was significantly shorter in patients with preablation AF than in patients without preablation AF.

This important issue has never been considered before and may change our current understanding of the clinical coexistence of typical AFL and AF. To date, electrophysiological mechanisms of coexisting AFL and AF in the same 
patient have focused on the dependence of one on the other. Nabar et $a l^{14}$ suggested that at least three different mechanisms may be responsible for this coexistence. More recently, on the basis of the important findings of Haissaguerre et al, ${ }^{27}$ Roithinger et $a l^{17}$ hypothesised that the persistence of rapidly firing foci may be the underlying mechanism for AF occurrence despite transisthmic ablation. Hsieh et al ${ }^{16}$ proceeded in the same direction and showed that atrial ectopic beats were able both to induce primary AF and to degenerate typical AFL into AF. Hence, at least in some patients, AF and AFL may be two different clinical expressions of the same electrical disease: focal arrhythmogenic triggers that persist despite elimination of AF, and that can, as time passes, induce AF once the preferential path through the flutter circuit is blocked. ${ }^{17-27}$

In some previous studies, postablation $\mathrm{AF}$ in patients without preablation AF had been reported to occur less frequently (from $8 \%$ to $12 \%$ ) than in our study. ${ }^{11-15}$ The reason for this discrepancy may lie in the different length of follow up, this study being the first with $45 \%$ of patients followed up for more than 24 months. In fact, we showed that the rate of AF occurrence in the subgroup of patients without preablation AF only increased dramatically after two years of follow up. Another reason for the higher occurrence of postablation AF is the systematic use of ECG Holter monitoring during our follow up, which led to the discovery of asymptomatic AF in 50 of 357 (14\%) patients.

\section{Variables correlated with AF occurrence}

Our data show that the occurrence of AF after transisthmic ablation is significantly correlated with preablation AF, with a younger age, and with a very enlarged left atrium. Previous history of concomitant AFL and AF and left atrial size had already been correlated with the occurrence of postablation AF by other authors. ${ }^{10-12141521}$ Preablation AF identifies patients in whom there is a structural and electrophysiological substrate that allows multi-reentrant circuits favouring AF. ${ }^{11}{ }^{12}$ Left atrial size is strictly correlated with AF: this arrhythmia is likely to occur in a more diseased, and thus enlarged, atrium. ${ }^{10}$ An absolute new finding is the inverse correlation of age with occurrence of postablation AF. According to the knowledge about AF prevalence in the general population, ${ }^{28}$ one would expect postablation AF to occur more frequently in the elderly. On the contrary, patients younger than 65 years experienced postablation AF more frequently than patients older than 65 years despite the similar rates of preablation $\mathrm{AF}$, predominant preablation $\mathrm{AF}$, and use of AADs in the two groups.

In previous studies, left ventricular ejection fraction and the inducibility of sustained AF after transisthmic ablation also proved to be predictors of postablation AF occurrence. ${ }^{8} 11121521$ In our study neither left ventricular ejection fraction nor the presence of structural heart disease predicted postablation AF, which may have been because in our population only $6 \%$ of patients had a depressed left ventricular function. Instead, we did not evaluate induction of sustained AF after ablation.

\section{Clinical implications}

Results from our study show that postablation AF occurs frequently and that its occurrence increases during follow up. This is true both for patients with preablation AF and for patients without. Indeed, after four years, the probability of postablation $\mathrm{AF}$ was $68 \%$ for patients with preablation $\mathrm{AF}$ and $52 \%$ for patients without preablation AF. This also means that the risk of the development of AF increases with time in patients with preablation lone AFL. Hence, they must be advised of the risk of recurrent symptoms and late AF and followed up closely despite successful transisthmic ablation.
ECG Holter monitoring must be advised too because up to $14 \%$ of AF episodes are asymptomatic.

Patients with preablation AF and an extremely enlarged left atrium are at very high risk of AF development despite continuation of AAD. Anticoagulation should be continued during the follow up because of the risk of stroke. However, transisthmic ablation should also be advised for these patients, as it reduces the total arrhythmic burden and is the first step towards future transcatheter treatment of AF. This kind of patient should be considered immediately for additional ablation procedures that eliminate triggers or modify the substrate. ${ }^{27} 29$

\section{Study limitations}

It is possible that in some patients with paroxysmal asymptomatic AF the occurrence of this arrhythmia during follow up is underestimated.

Although bidirectional transisthmic block is considered the criterion for successful ablation in the literature, we also used unidirectional block as a criterion defining successful ablation because at the beginning of the study it was an accepted criterion in several of the participating centres.

AADs were advised for all patients with preablation AF. Indeed, our results on postablation AF occurrence do not reflect the pure effect of transisthmic ablation but rather the combination of pharmacological and ablative treatments. ${ }^{14}$

Inducibility of sustained AF after transisthmic ablation was not considered among the variables that may correlate with postablation AF occurrence because different protocols of atrial stimulation were used in the study patients.

\section{Authors' affiliations}

E Bertaglia, F Zoppo, D D'Este, F Zerbo, P Pascotto, Departments of Cardiology, Ospedale Civile, Mirano, Italy

A Bonso, Ospedale Umberto I, Mestre, Italy

A Proclemer, Ospedale S Maria della Misericordia, Udine, Italy

R Verlato, Ospedale Civile, Camposampiero, Italy

L Corò, Ospedale Civile, Conegliano, Italy

R Mantovan, Ospedale Ca'Foncello, Treviso, Italy

A complete list of investigators appears in the appendix.

\section{APPENDIX}

The following centres and investigators participated in the study (number of patients in parentheses):

- Ospedale Civile, Mirano: E Bertaglia, D D’Este, P Pascotto, F Zerbo, F Zoppo, (110);

- Ospedale Umberto I, Mestre: A Bonso, A Corrado, A Raviele, A Rossillo, S Temistoklakis (80);

- Ospedale S Maria della Misericordia, Udine: M Carone, D Facchin, A Proclemer (65);

- Ospedale Civile, Camposampiero: M S Baccellieri, R Pastore, P Turrini, R Verlato (55);

- Ospedale Civile, Conegliano: L Corò, N Sitta, P Delise (40);

- Ospedale Ca'Foncello, Treviso: V Calzolari, R Mantovan, E Marras (33).

Coordinators: E Bertaglia, A Bonso.

\section{REFERENCES}

1 Cosio FG, Lopez-Gil M, Giocolea A, et al. Radiofrequency ablation of the inferior cava-tricuspid valve isthmus in common atrial flutter. Am J Cardiol 1993:71:705-9.

2 Fischer B, Haissaguerre M, Garrigues S, et al. Radiofrequency catheter ablation of common atrial flutter in 80 patients. J Am Coll Cardiol 1995;25:1365-72.

3 Lee SH, Tai CT, Yu WC, et al. Effects of radiofrequency catheter ablation on quality of life in patients with atrial flutter. Am J Cardiol 1999;84:278-83. 
4 Natale A, Newby KH, Pisanò E, et al. Prospective randomized comparison of antiarrhythmic therapy versus first-line radiofrequency ablation in patients with atrial flutter. J Am Coll Cardiol 2000;35:1898-904.

5 Poty H, Saoudi N, Aziz AA, et al. Radiofrequency catheter ablation of type 1 atrial flutter: prediction of late success by electrophysiological criteria. Circulation 1995;92:1389-92.

6 Shah DC, Takahashi A, Jais P, et al. Local electrogram-based criteria of cavotricuspid isthmus block. J Cardiovasc Electrophysiol 1999:10:662-9.

7 Anselme $F$, Savourè A, Cribier A, et al. Catheter ablation of typical atrial flutter. A randomized comparison of 2 methods for determining complete bidirectional isthmus block. Circulation 2001;103:1434-9.

8 Philippon F, Plumbe VJ, Epstein A, et al. The risk of atrial fibrillation following radiofrequency catheter ablation of common atrial flutter. Circulation 1995;92:430-5.

9 Saxon LA, Kalman JM, Olgin JE, et al. Results of radiofrequency catheter ablation for atrial flutter. Am J Cardiol 1996;77:1014-6.

10 Frey B, Kreiner B, Binder T, et al. Relation between left atrial size and secondary atrial arrhythmias after successful catheter ablation of common atrial flutter. Pacing Clin Electrophysiol 1997;20:2936-42.

11 Tai CT, Chen SA, Chiang CE, et al. Long term outcome of radiofrequency catheter ablation for typical atrial flutter: risk prediction of recurrent arrhythmias. J Cardiovasc Electrophysiol 1998;9:115-21.

12 Paydak H, Kall JG, Burke MC, et al. Atrial fibrillation after radiofrequency ablation of type I atrial flutter: time to onset, determinants and clinical course. Circulation 1998;98:315-22

13 Anselme F, Saoudi N, Poty H, et al. Radiofrequency catheter ablation of common atrial flutter: significance of palpitations and quality of life evaluation in patients with proven isthmus block. Circulation 1999;99:534-40.

14 Nabar A, Rodriguez LM, Timmermans C, et al. Effect of right atrial isthmus ablation on the occurrence of atrial fibrillation. Observations in four patient groups having type I atrial flutter with or without associated atrial fibrillation. Circulation 1999;99:1441-5.

15 Da Costa A, Romeyer C, Mourot S, et al. Factors associated with early atrial fibrillation after ablation of common atrial flutter. A single centre prospective study. Eur Heart J 2002;23:498-506.
16 Hsieh MH, Tai CT, Tsai CF, et al. Mechanism of spontaneous transition from typical atrial flutter to atrial fibrillation. Pacing Clin Electrophysiol $2001 ; 24: 46-52$

17 Roithinger FX, Lesh MD. What is the relationship of atrial flutter and fibrillation? Pacing Clin Electrophysiol 1999;22:643-54.

18 Huang DT, Monahan KM, Papageorgiou P, et al. Hybrid pharmacologic and ablative therapy: a novel and effective approach for the management of atrial fibrillation. J Cardiovasc Electrophysiol 1998;9:462-9.

19 Schumacher B, Jung W, Lewalter T, et al. Radiofrequency ablation of atrial flutter due to administration of class IC antiarrhythmic drugs for atrial fibrillation. Am J Cardiol 1999;83:710-3.

20 Reithmann C, Hoffmann E, Spitzberger G, et al. Catheter ablation of atrial flutter due to amiodarone therapy for paroxysmal atrial fibrillation. Eur Heart $J$ 2000;21:565-72.

21 Hsieh MH, Tai CT, Chiang CE, et al. Recurrent atrial flutter and atrial fibrillation after catheter ablation of the cavotricuspid isthmus: a very longterm follow-up of 333 patients. J Interv Card Electrophysiol 2002;7:225-31.

22 Folland E, Parisi A, Moynihan $\mathrm{P}$, et al. Assessment of left ventricular ejection fraction and volume by real-time, two-dimensional echocardiography. Circulation 1979;60:760-6.

23 Poty H, Saoudi N, Nair M, et al. Radiofrequency catheter ablation of atrial flutter: further insights into the various types of isthmus block: application to ablation during sinus rhythm. Circulation 1996:94:3204-13.

24 Kaplan EL, Meier P. Non parametric estimation from incomplete observations. $J$ Am Stat Assoc 1958;53:457-81.

25 Savage IR. Contributors to the theory of rank order statistics: the two sample case. Ann Math Stat 1956;27:590-615.

26 Cox DR. Regression models and life-tables. J R Stat Soc $[B]$ 1972;34:187-220

27 Haissaguerre $M$, Jais $P$, Shah DC, et al. Spontaneous initiation of atrial fibrillation by ectopic beats originating in the pulmonary veins. N Engl J Med 1998;339:659-66

28 Onundarson PT, Thorgeirsson G, Jonmundsson E, et al. Chronic atrial fibrillation: epidemiologic features and 14 years follow-up: a case-control study. Eur Heart J 1987;8:521-7.

29 Pappone C, Rosanio S, Oreto G, et al. Circumferential radiofrequency ablation of pulmonary vein ostia. Circulation 2000;102:2619-28.

\section{IMAGES IN CARDIOLOGY}

\section{Echocardiographic diagnosis of cor triatriatum sinister in the adult}
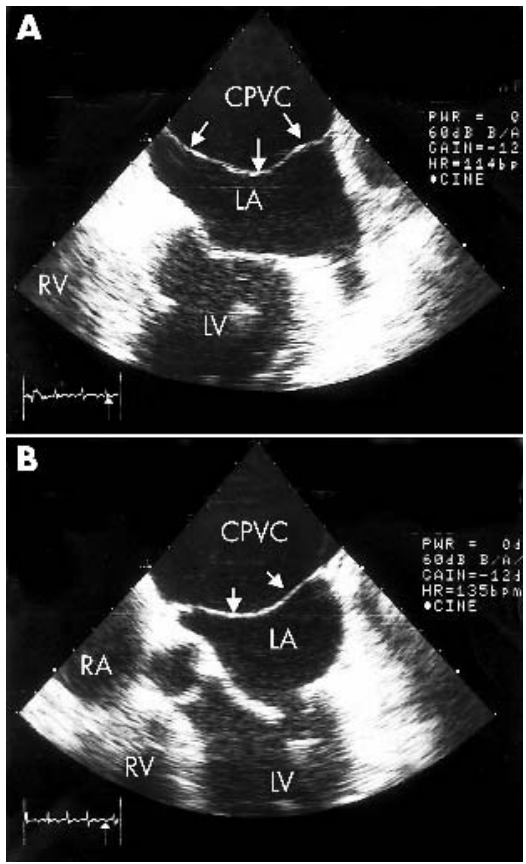

Transoesophageal echocardiography in systole (A) and diastole (B) demonstrating the left atrial membrane (white arrows). CPVC, common pulmonary venous chamber; $L A$, true left atrium; LV, left ventricle; RV, right ventricle; RA, right atrium.
46 year old man presented with a short history of flu like symptoms and the clinical finding of atrial fibrillation. Transthoracic echocardiography raised the suspicion of a poorly defined membrane within the left atrium. Transoesophageal echocardiography demonstrated a thick membrane dividing the left atrium into a common pulmonary venous chamber, receiving the pulmonary veins, and the true left atrium (panels A and B). This was attached medially to the margin of the fossa ovalis and laterally to the junction of the left superior pulmonary vein and the left atrial appendage. Doppler studies estimated the mean trans membrane pressure gradient at $2 \mathrm{~mm} \mathrm{Hg}$. The images were consistent with the diagnosis of cor triatriatum sinister.

Surgical excision of the left atrial membrane was performed and the patient made a full recovery.

\section{R D Slight}

O C Nzewi

P S Mankad

rdslight@btopenworld.com 